\title{
Localisation Inverse Problem and Dirichlet-to-Neumann Operator for Absorbing Laplacian Transport
}

\author{
Ibrahim Baydoun \\ École centrale Paris, Laboratoire de MSS-MAT, F-92295 Grande voie des Vignes, Châtenay-Malabry cedex, France \\ Email: ibrahim.baydoun@ecp.fr \\ Received March 18, 2013; revised April 18, 2013; accepted May 13, 2013
}

Copyright (C) 2013 Ibrahim Baydoun. This is an open access article distributed under the Creative Commons Attribution License, which permits unrestricted use, distribution, and reproduction in any medium, provided the original work is properly cited.

\begin{abstract}
We study Laplacian transport by the Dirichlet-to-Neumann formalism in isotropic media $(\gamma=I)$. Our main results concern the solution of the localisation inverse problem of absorbing domains and its relative Dirichlet-to-Neumann operator $\Lambda_{\gamma=I, \partial \Omega}$. In this paper, we define explicitly operator $\Lambda_{\gamma=I, \partial \Omega}$, and we show that Green-Ostrogradski theorem is adopted to this type of problem in three dimensional case.
\end{abstract}

Keywords: Absorbing Laplacian Transport; Dirichlet-to-Neumann Operators; Inverse Problem

\section{Laplacian Transport and Dirichlet-to-Neumann Operators}

The theory of Dirichlet-to-Neumann operators is the basis of many research domains in analysis, particularly, those concerning Laplacian transports. It is also very important in mathematical-physics, geophysics, electrochemistry. Moreover, it is very useful in medical diagnosis, such as electrical impedance tomography:

In 1989, J. Lee and G. Uhlmann have introduced an example on the determination of conductivity matrix field in a bounded open domain, see e.g. [1]. This example is related to measuring the elliptic Dirichlet-toNeumann map for associated conductivity equation, see e.g. [1].

The problem of electrical current flux is an example of so-called diffusive Laplacian transport. Besides the voltage-to-current problem, the motivation to study this kind of transport comes for instance, from the transfer across biological membranes, see e.g. [2,3].

Let some species of concentration $v(p), \quad x \in \mathbb{R}^{d}$, diffuse stationary in the isotropic bulk $(\gamma=I)$ from a (distant) source localised on the closed boundary $\partial \Omega$ towards a semipermeable compact interface $\partial B$ of the cell $\bar{B} \subset \Omega$, where they disappear at a given rate $W \geq 0$. Then the steady field of concentrations (Laplacian transport with a diffusion coefficient $D \geq 0$ ) obeys the set of equations:

$$
\text { (P1) }\left\{\begin{array}{l}
\Delta v=0, p \in \Omega \backslash \bar{B} \\
\left.v\right|_{\partial \Omega}(p)=f(p), \text { the concentration at the source } \partial \Omega, \\
-\left.D \partial_{v} v\right|_{\partial B}(\omega)=\left.W\left(v-c^{*}\right)\right|_{\partial B}(\omega), \text { on the interface } \omega \in \partial B .
\end{array}\right.
$$

Usually, one supposes that $v(p)=c^{*} \geq 0, \quad p \in B$, is a constant concentration of the species inside the cell $\bar{B}$. This example motivates the following abstract stationary diffusive Laplacian transport problem with absorption on the surface $\partial B$ :

(P2)

$$
\left\{\begin{array}{l}
\Delta v=0, p \in \Omega \backslash \bar{B},[v(p)=\text { Const, } p \in \bar{B}] \\
\left.v\right|_{\partial \Omega}(p)=f(p), p \in \partial \Omega \\
\left.\left(\alpha v+\partial_{v} v\right)\right|_{\partial B}(\omega)=h(\omega), \omega \in \partial B
\end{array}\right.
$$

This is the Dirichlet-Neumann problem for domain $\Omega \supset \bar{B}$ with the Robin [4] boundary condition on the absorbing surface $\partial B$. Varying $\alpha:=W D^{-1}$ between $\alpha=0$ and $\alpha=+\infty$, one recovers respectively the Neumann and the Dirichlet boundary conditions.

Now, we can associate with the problem (P2) a Dirihlet-to-Neumann operator

$$
\Lambda_{\gamma=I, \partial \Omega}: f \mapsto g:=\left.\partial_{\nu} v_{f}\right|_{\partial \Omega} .
$$

Domain $\operatorname{dom}\left(\Lambda_{I, \partial \Omega}\right)$ belongs to a certain Sobolev 
space of functions on the boundary $\partial \Omega$, which contains $v_{f}:=v_{f}^{(\alpha, g)}$, the solutions of the problem (P2) for given $f$ and for the Robin boundary condition on $\partial B$ fixed by $\alpha$ and $g$.

The advantage of this approach is that as soon as the operator (1) is defined, one can apply it to study the mixed boundary value problem (P2). This gives, in particularly, the value of the particle flux due to Laplacian transport across the membrane $\partial \Omega$. Moreover, the total current across the boundary $\partial \Omega$ can be defined (for given $f$ ) in term of Dirihlet-to-Neumann operator (1) as follows:

$$
J_{\partial \Omega}:=-D \int_{\partial \Omega} \mathrm{d} \sigma \Lambda_{\gamma=I, \partial \Omega} f,
$$

where $\mathrm{d} \sigma$ designed the differential element relative to $\partial \Omega$.

There are at least two inverse problems derived from problem (P2):

a) geometrical inverse problem: given Dirichlet data $f$ and the corresponding (measured) Neumann data $g$, in (1), on the accessible outer boundary $\partial \Omega$, to reconstruct the shape of the interior boundary $\partial B$, see [5].

b) localisation inverse problem: concerns to localisate of the domain (cell) $\bar{B}$ with a given shape and the fixed parameters $\alpha$ and $h$, see [6].

The main question in this context is to find sufficient conditions insuring that the localization inverse problem is uniquely soluble. Indeed:

First, we relate the above problems a) and b) with the Dirichlet-to-Neumann operator (1) by defining explicitly this operator, whose can define the local and total current across the external boundary $\partial \Omega$, which are useful to resolve a) and b).

Second, we study the localisation inverse problem in the framework of application outlined in the problem (P2), which consist in finding sufficient (Dirichlet-toNeumann) conditions to localise the position of the cell $\bar{B}$ from the experimentally measurable macroscopic response parameters.

In Section 2, we introduce the existence and uniqueness for the solution of problem (P2). In Section 3, we introduce our first main result concerning the study of spherical case of problem (P1), whose we give a general method to resolve the type of partial derivative system like (P1), see proposition 3.2. Indeed, we allow an explicit calculations, based on Green-Ostrogradski theorem, for the solution of this problem.

In Section 4, it is our second main result which consist in showing that total current across the external boundary $\partial \Omega$, involving Dirihlet-to-Neumann operator (1), can resolve the localisation inverse problem in three dimensional case, when the compact $\Omega \subset \mathbb{R}^{3}$.

\section{Uniqueness of the Problem (P2)}

We suppose that $\Omega$ and $B \subset \Omega$ be open bounded domains in $\mathbb{R}^{d}$ with $C^{2}$-smooth disjoint boundaries $\partial \Omega$ and $\partial B$, that is $\partial(\Omega \backslash \bar{B})=\partial \Omega \cup \partial B$ and $\partial \Omega \cap \partial B=\varnothing$.

Then the unit outer-normal to the boundary $\partial(\Omega \backslash \bar{B})$ vector-field $v(x)_{x \in \partial(\Omega \backslash B)}$ is well-defined, and we consider the normal derivative in (P2) as the interior limit:

$$
\left.\left(\partial_{\nu} u\right)\right|_{\partial в}(\omega):=\lim _{x \rightarrow \omega} v(\omega) \cdot(\nabla u)(x), x \in \Omega \backslash \bar{B} .
$$

The existence of the limit (3) as well as the restriction $\left.u\right|_{\partial B}(\omega):=\lim _{x \rightarrow \infty} \mu(x)$ is insured since $u$ has to be harmonic solution of problem (P2) for $C^{2}$-smooth boundaries $\partial(\Omega \backslash \bar{B})$ [7].

Now, we introduce some indispensable standard notations and definitions, see [8]. Let $\mathcal{H}$ be Hilbert space $L^{2}(M)$ on domain $M \subset \mathbb{R}^{d}$ and $\partial \mathcal{H}:=L^{2}(\partial M)$ denote the corresponding boundary space. We denote by $W_{2}^{s}(M)$ the Sobolev space of $\mathcal{H}$-functions, whose $s$ derivatives are also in $\mathcal{H}$, and similar, $W_{2}^{s}(\partial M)$ is the Sobolev space of $\partial \mathcal{H}$-functions on the $C^{2}$-smooth boundary $\partial M$.

Proposition 2.1. Let $f \in W_{2}^{1 / 2}(\partial \Omega)$ for $C^{2}$-smooth boundaries $\partial(\Omega \backslash \bar{B})$. Then the Dirichlet-Neumann problem (P2) has a unique (harmonic) solution in domain $\Omega \backslash \bar{B}$.

Proof. For existence we refer to [7]. To prove the uniqueness, we consider the problem (P2) for $f=0$ and $c^{*}=0$. Then by Gauss-Ostrogradsky theorem, one gets that the corresponding solution $u$ yields:

$$
\begin{aligned}
& \int_{\Omega \backslash \bar{B}} \mathrm{~d} x(\nabla \overline{u(x)} \cdot \nabla u)(x) \\
& =\int_{\Omega \backslash \bar{B}} \mathrm{~d} x \operatorname{div}(\overline{u(x)}(\nabla u)(x)) \\
& =\int_{\partial B} \mathrm{~d} \sigma(\omega) \overline{u(\omega)}\left(\partial_{v} u\right)(\omega) \\
& =-W D^{-1} \int_{\partial B} \mathrm{~d} \sigma(\omega)|u(\omega)|^{2} \leq 0 .
\end{aligned}
$$

The estimate (4) implies that $u(x \in \Omega \backslash \bar{B})=$ Const . Hence by the boundary condition one gets $\left.\left(W D^{-1} u\right)\right|_{\partial B}(\omega)=0$, and from $\left.u\right|_{\partial \Omega}(x)=f(x \in \partial \Omega)=0$, we obtain that for $W D^{-1} \geq 0$, the harmonic function $u(x)=0$ for $x \in \Omega \backslash \bar{B}$. $\square$

The next statement is a key for analysis of inverse localisation problems:

Proposition 2.2. Consider two problems (P2) corresponding to a bounded domain $\Omega \subset \mathbb{R}^{2}$ with $C^{2}$ smooth boundary $\partial \Omega$ and to two subsets $B_{1}$ and $B_{2}$ with the same smoothness of the boundaries $\partial B_{1}, \partial B_{2}$. If for solutions $u_{f}^{(1)}, u_{f}^{(2)}$ of these problems one has 


$$
\left.\partial_{\nu} u_{f}^{(1)}\right|_{\partial \Omega}=\left.\partial_{\nu} u_{f}^{(2)}\right|_{\partial \Omega},
$$

then $\partial B_{1}=\partial B_{2}$.

Proof. By virtue of $\left.u_{f}^{(1)}\right|_{\partial \Omega}=\left.u_{f}^{(2)}\right|_{\partial \Omega}=f$ and by condition (5), the problem (P2) has two solutions for identical external (on $\partial \Omega$ ) and internal (on $\partial B_{1}$ and $\partial B_{2}$ ) Robin boundary conditions. Then by the standard arguments based on the Holmgren uniqueness theorem [9] for harmonic functions on $\mathbb{R}^{2}$, one obtains that $\partial B_{1}=\partial B_{2}$.

\section{Dirichlet-to-Neumann Operators for Absorbing Laplacian Transport}

Here, we consider the spherical shell of the problem (P1) so that $\Omega=B\left(O_{0}, R_{0}\right)$ and the absorbing cell is also a ball $B=B\left(O, r_{0}<R_{0}\right)$, whose we denote by $d_{0}$ the distance between the two centers $d_{0}=d_{O_{0} \rightarrow O}$.

Hereafter, we denote the previous hypothesis by spherical case.

In the sequel, we resolve the problem (P1) in order to calculate explicitly Dirichlet-to-Neumann operator relative to this case.

Before resolving problem (P1), we need the following theorem which the key of the solution:

Theorem 3.1. (Gauss-Ostrogradski)
Let $\boldsymbol{V}$ a field vector across the domain $\Psi \subset \mathbb{R}^{3}$, having as border $\partial \Psi$.

$$
\int_{M \in \partial \Psi} \boldsymbol{V} \cdot \boldsymbol{n}_{M} \mathrm{~d} \sigma=\int_{\Psi} \operatorname{div} \boldsymbol{V} \mathrm{d} \Psi,
$$

whose $\operatorname{div} \boldsymbol{V}$ designated the divergence of field vector $\boldsymbol{V} . \mathrm{d} \sigma$ and $\mathrm{d} \Psi$ designated respectively the differential elements relative to $\partial \Psi$ and $\Psi . n_{M}$ designated the unit outer-normal vector on $\partial \Psi$ at arbitrary point $M$.

Remark 1. Let the orthonormal reference with origin $O$ and axis $Y^{\prime} Y$, which is keen on the line $O O_{0}$ in the sense of the vector $\mathrm{OO}_{0}$.

On the other hand, since for all $l \in \mathbb{N}$, spherical harmonic function $Y_{l, m=0}(\theta, \varphi)$ is independent of $\varphi$ if $m=0$, then we note:

$$
Y_{l}(\theta):=Y_{l, m=0}(\theta, \varphi) .
$$

Since $v_{f}$ is harmonic function, then it takes the following form, see [10]:

$$
v_{f}(r, \theta, \varphi)=\sum_{l=0}^{\infty} \sum_{m=-l}^{l}\left(a_{l} r^{l}+b_{l} r^{-l-1}\right) Y_{l, m}(\theta, \varphi) .
$$

Therefore, we need to calculate the coefficients of (7) from the condition boundaries. Indeed, since the radius of points of $\partial B$ are equal to constant $r_{0}$, then the condition boundary on $\partial B$ implies easily, by identification, the following system:

$$
\left(S_{f}\right) \begin{cases}a_{0}=-b_{0}\left(D W^{-1} r_{0}^{-2}+r_{0}^{-1}\right)+c^{*} Y_{0,0}^{-1}(\varphi, \theta) & \text { ifl }=0, \\ a_{l}=b_{l}\left[D(l+1) r_{0}^{-l-2}+W r_{0}^{-l-1}\right]\left(D l r_{0}^{l-1}-W r_{0}^{l}\right)^{-1} & i f l \neq 0 .\end{cases}
$$

But, on the boundary $\partial \Omega$, the radius aren't equal, and depend of spherical angle $\theta$. Then, for this reason, we use Gauss-Ostrogradski theorem's, whose we show that it is useful to find another relation between the coefficients of (7) like $\left(S_{f}\right)$. Consequently, we get for each

$$
\int_{0}^{2 \pi} \int_{0}^{\pi} \mathrm{d} \varphi \mathrm{d} \theta \sin \theta Y_{l}(\theta)\left[f(\varphi, \theta) r^{l+1}(\theta)-a_{l} r^{2 l+1}(\theta) Y_{l}(\theta)\right]=b_{l}, \forall l \in \mathbb{N} .
$$

Proof. Let $l_{0} \in \mathbb{N}$, we construct the following vector field $V_{l_{0}}$ by:

$$
\boldsymbol{V}_{l_{0}}=H_{l_{0}}(r, \varphi, \theta) \boldsymbol{e}_{\varphi},
$$

whose, $H_{l_{0}}(r, \varphi, \theta)$ is a primitive relative to $\varphi$ for the following function:

$$
h_{l_{0}}(r, \varphi, \theta)=Y_{l_{0}}(\theta) \frac{\sin \theta}{r}\left[\partial_{r}\left(\frac{v(r, \varphi, \theta)-a_{l_{0}} r^{l_{0}} Y_{l_{0}}(\theta)}{b_{l_{0}} r^{-l_{0}-1}}\right)\right] \text {. }
$$

$l \in \mathbb{N}$, a system of two equations with two unknowns $a_{l}$ and $b_{l}$, which it is sufficient to calculate $a_{l}$ and $b_{l}$ :

Proposition 3.2. The condition boundary on $\partial \Omega \mathrm{im}$ plies:

$$
\begin{cases}\boldsymbol{n} & \text { unit outer-normal vector of } \partial\left[B\left(O_{0}, R_{0}\right) \backslash B\left(O, r_{0}\right)\right], \\ \mathrm{d} \sigma & \text { areal differential elemen trelative to } \partial\left[B\left(O_{0}, R_{0}\right) \backslash B\left(O, r_{0}\right)\right], \\ \mathrm{d} v=r^{2} \sin \theta \mathrm{d} r \mathrm{~d} \varphi \mathrm{d} \theta & \text { volume differential elemen trelative to } B\left(O_{0}, R_{0}\right) \backslash B\left(O, r_{0}\right), \\ \operatorname{div}(\boldsymbol{v}) & \text { divergence of vector } \boldsymbol{v} .\end{cases}
$$

Calculate the flux of field $\boldsymbol{V}_{l_{0}}$ across the domain $B\left(O_{0}, R_{0}\right) \backslash B\left(O, r_{0}\right)$ using Gauss-Ostrogradski theorem 3.1:

$$
\begin{aligned}
& \int_{B\left(O_{0}, R_{0}\right) \backslash B\left(O, r_{0}\right)} \mathrm{d} v \operatorname{div}\left(\boldsymbol{V}_{l_{0}}\right) \\
& =\int_{\partial\left[B\left(O_{0}, R_{0}\right) \backslash B\left(O, r_{0}\right)\right]} \boldsymbol{V}_{l_{0}} \cdot \boldsymbol{n d} \sigma,
\end{aligned}
$$

where: 
1. Calculate $\int_{B\left(O_{0}, R_{0}\right) \backslash B\left(O, r_{0}\right)} \operatorname{dvdiv}\left(\boldsymbol{V}_{l_{0}}\right)$ :

In domain $B\left(O_{0}, R_{0}\right) \backslash B\left(O, r_{0}\right)$, we have:

Radius $r$ varies between $r_{0}$ and $r(\theta):=r_{M \in \partial B\left(O_{0}, R_{0}\right)}$.

Angle $\varphi$ varies between 0 and $2 \pi$.

Angle $\theta$ varies between 0 and $\pi$.
On the other hand, $\operatorname{div}\left(\boldsymbol{V}_{l_{0}}\right)$ can be calculated from (9) by:

$$
\begin{aligned}
& \operatorname{div}\left(\boldsymbol{V}_{l_{0}}\right)=\frac{1}{r \sin \theta} \partial_{\varphi} H(r, \varphi, \theta) \\
& =\frac{Y_{l_{0}}(\theta)}{r^{2}} \partial_{r}\left(\frac{v(r, \varphi, \theta)-a_{l_{0}} r^{l_{0}} Y_{l_{0}}(\theta)}{b_{l_{0}} r^{-l_{0}-1}}\right) .
\end{aligned}
$$

Then, we deduce that:

$$
\begin{aligned}
\int_{B\left(O_{0}, R_{0}\right) \backslash B\left(O, r_{0}\right)} \operatorname{d} v \operatorname{div}\left(\boldsymbol{V}_{l_{0}}\right) & =\int_{r_{0}}^{r(\theta)} \int_{0}^{2 \pi} \int_{0}^{\pi} r^{2} \sin \theta \mathrm{d} r \mathrm{~d} \varphi \mathrm{d} \theta \operatorname{div}\left(\boldsymbol{V}_{l_{0}}\right) \\
& =\int_{r_{0}}^{r(\theta)} \int_{0}^{2 \pi} \int_{0}^{\pi} \mathrm{d} r \mathrm{~d} \varphi \mathrm{d} \theta \sin \theta Y_{l_{0}}(\theta) \partial_{r}\left(\frac{v(r, \varphi, \theta)-a_{l_{0}} r^{l_{0}} Y_{l_{0}}(\theta)}{b_{l_{0}} r^{-l_{0}-1}}\right) .
\end{aligned}
$$

Therefore, from Fubini's theorem of multiple integrals, we obtain:

$$
\begin{aligned}
& \int_{B\left(O_{0}, R_{0}\right) \backslash B\left(O, r_{0}\right)} \mathrm{d} v \operatorname{div}\left(\boldsymbol{V}_{l_{0}}\right) \\
& =\int_{0}^{2 \pi} \int_{0}^{\pi} \mathrm{d} \varphi \mathrm{d} \theta \sin \theta Y_{l_{0}}(\theta) \int_{r_{0}}^{r(\theta)} \partial_{r}\left[\frac{v(r, \varphi, \theta)-a_{l_{0}} r^{l_{0}} Y_{l_{0}}(\theta)}{b_{l_{0}} r^{l_{0}-1}}\right] \mathrm{d} r \\
& =\int_{0}^{2 \pi} \int_{0}^{\pi} \mathrm{d} \varphi \mathrm{d} \theta \sin \theta Y_{l_{0}}(\theta)\left[\frac{v(r, \varphi, \theta)-a_{l_{0}} r^{l_{0}} Y_{l_{0}}(\theta)}{b_{l_{0}} r^{-l_{0}-1}}\right] r(\theta) \\
& =\frac{1}{b_{l_{0}}} \int_{0}^{2 \pi} \int_{0}^{\pi} \mathrm{d} \varphi \mathrm{d} \theta \sin \theta Y_{l_{0}}(\theta)\left[v(r(\theta), \varphi, \theta) r^{l_{0}+1}(\theta)-a_{l_{0}} r^{2 l_{0}+1}(\theta) Y_{l_{0}}(\theta)\right] \\
& -\frac{1}{b_{l_{0}}} \int_{0}^{2 \pi} \int_{0}^{\pi} \mathrm{d} \varphi \mathrm{d} \theta \sin \theta Y_{l_{0}}(\theta) r_{0}^{l_{0}+1}\left[v\left(r_{0}, \varphi, \theta\right)-a_{l_{0}} r_{0}^{l_{0}} Y_{l_{0}}(\theta)\right] .
\end{aligned}
$$

Moreover, condition boundary on $\partial \Omega$ implies that:

$$
v(r(\theta), \varphi, \theta)=v\left[r_{M \in \partial B\left(O_{0}, R_{0}\right)}, \varphi, \theta\right]=v\left[M \in \partial B\left(O_{0}, R_{0}\right)\right]=f(\varphi, \theta) .
$$

So, by replacing $v(r(\theta), \varphi, \theta)$ by its value $f(\varphi, \theta)$ in (11), we deduce:

$$
\begin{aligned}
& \int_{B\left(O_{0}, R_{0}\right) \backslash B\left(O, r_{0}\right)} \mathrm{d} v \operatorname{div}\left(\boldsymbol{V}_{l_{0}}\right) \\
& =\frac{1}{b_{l_{0}}} \int_{0}^{2 \pi} \int_{0}^{\pi} \mathrm{d} \varphi \mathrm{d} \theta \sin \theta Y_{l_{0}}(\theta)\left[f(\varphi, \theta) r^{l_{0}+1}(\theta)-a_{l_{0}} r^{2 l_{0}+1}(\theta) Y_{l_{0}}(\theta)\right] \\
& -\frac{1}{b_{l_{0}}} \int_{0}^{2 \pi} \int_{0}^{\pi} \mathrm{d} \varphi \mathrm{d} \theta \sin \theta Y_{l_{0}}(\theta) r_{0}^{l_{0}+1}\left[v\left(r_{0}, \varphi, \theta\right)-a_{l_{0}} r_{0}^{l_{0}} Y_{l_{0}}(\theta)\right] .
\end{aligned}
$$

But, we can prove that:

$$
\int_{0}^{2 \pi} \int_{0}^{\pi} \mathrm{d} \varphi \mathrm{d} \theta \sin \theta Y_{l_{0}}(\theta) r_{0}^{l_{0}+1}\left[v\left(r_{0}, \varphi, \theta\right)-a_{l_{0}} r_{0}^{l_{0}} Y_{l_{0}}(\theta)\right]=b_{l_{0}} .
$$

Indeed: from (7), we have that

$$
r_{0}^{l_{0}+1}\left[v\left(r_{0}, \varphi, \theta\right)-a_{l_{0}} r_{0}^{l_{0}} Y_{l_{0}}(\theta)\right]=\sum_{\substack{l=0 \\ l \neq l_{0}}}^{+\infty} \sum_{m=-l}^{m=l}\left(a_{l} r_{0}^{l+l_{0}+1}+b_{l} r_{0}^{-l+l_{0}}\right) Y_{l, m}(\varphi, \theta)+b_{l_{0}} Y_{l_{0}}(\theta)
$$


Multiplying by $\sin \theta$ the previous equation, and integrating it on domain $\{(\varphi, \theta): \varphi \in[0,2 \pi[, \theta \in[0, \pi[\}$, we obtain:

$$
\begin{aligned}
& \int_{0}^{2 \pi} \int_{0}^{\pi} \mathrm{d} \varphi \mathrm{d} \theta \sin \theta Y_{l_{0}}(\theta) r_{0}^{l_{0}+1}\left[C\left(r_{0}, \varphi, \theta\right)-a_{l_{0}} r_{0}^{l_{0}} Y_{l_{0}}(\theta)\right] \\
& =b_{l_{0}} \int_{0}^{2 \pi} \int_{0}^{\pi} \mathrm{d} \varphi \mathrm{d} \theta \sin \theta Y_{l_{0}}(\theta)+\sum_{\substack{l=0 \\
l \neq l_{0}}}^{m} \sum_{m=-l}^{m=l}\left(a_{l} r_{0}^{l+l_{0}+1}+b_{l} r_{0}^{-l+l_{0}}\right) \int_{0}^{2 \pi} \int_{0}^{\pi} \mathrm{d} \varphi \mathrm{d} \theta \sin \theta Y_{l_{0}}(\theta) Y_{l, m}(\varphi, \theta) .
\end{aligned}
$$

On the other hand, spherical harmonic functions form a basis for the Hilbert space $L^{2}[S(O, 1)]$ following inner product:

$$
\begin{gathered}
\langle f, g\rangle:=\int_{S(O, 1)} \sin \theta \mathrm{d} \theta \mathrm{d} \varphi f \bar{g} ; \forall f, g \in L^{2}[S(O, 1)] . \quad \forall(l, m),(\tilde{l}, \tilde{m}) \\
\left\langle Y_{l_{0}}, Y_{l, m}\right\rangle:=\int_{0}^{2 \pi} \int_{0}^{\pi} \mathrm{d} \varphi \mathrm{d} \theta \sin \theta Y_{l_{0}}(\theta) Y_{l, m}(\varphi, \theta)=\delta_{l_{0}}(l) ; \quad \forall l, l_{0} \in \mathbb{N} .
\end{gathered}
$$

Consequently, we deduce, since

$$
\begin{aligned}
& \left\langle Y_{l, m}, Y_{\tilde{l}, \tilde{m}}\right\rangle=\delta_{(l, m)}(\tilde{l}, \tilde{m}) ; \\
& \forall(l, m),(\tilde{l}, \tilde{m}) \in \mathbb{N}
\end{aligned}
$$

Here, $\delta$ designed Dirac function.

So, by inserting (15) in (14), we deduce above equality (13) as follows:

$$
\begin{aligned}
\int_{0}^{2 \pi} \int_{0}^{\pi} \mathrm{d} \varphi \mathrm{d} \theta \sin \theta Y_{l_{0}}(\theta) r_{0}^{l_{0}+1}\left[v\left(r_{0}, \varphi, \theta\right)-a_{l_{0}} r_{0}^{l_{0}} Y_{l_{0}}(\theta)\right] & =\sum_{\substack{l=0 \\
l \neq l_{0}}}^{+\infty}\left(a_{l} r_{0}^{l+l_{0}+1}+b_{l} r_{0}^{-l+l_{0}}\right) \delta_{l_{0}}(l)+b_{l_{0}} \delta_{l_{0}}\left(l_{0}\right) \\
& =\sum_{\substack{l=0 \\
l \neq l_{0}}}^{+\infty}\left(a_{l} r_{0}^{l+l_{0}+1}+b_{l} r_{0}^{-l+l_{0}}\right) 0+b_{l_{0}} 1=b_{l_{0}} .
\end{aligned}
$$

We continue the proof by inserting (13) in (12):

$$
\int_{B\left(O_{0}, R_{0}\right) \backslash B\left(O, r_{0}\right)} \operatorname{dvdiv}\left(\boldsymbol{V}_{l_{0}}\right)=\frac{1}{b_{l_{0}}} \int_{0}^{2 \pi} \int_{0}^{\pi} \mathrm{d} \varphi \mathrm{d} \theta \sin \theta Y_{l_{0}}(\theta)\left[f(\varphi, \theta) r^{l_{0}+1}(\theta)-a_{l_{0}} r^{2 l_{0}+1}(\theta) Y_{l_{0}}(\theta)\right]-1 .
$$

2. Calculate $\int_{\partial\left[B\left(O_{0}, R_{0}\right) \backslash B\left(O, r_{0}\right)\right]} \boldsymbol{V}_{l_{0}} \cdot \boldsymbol{n} \mathrm{d} \sigma:$

knowing that,

$$
\partial\left[B\left(O_{0}, R_{0}\right) \backslash B\left(O, r_{0}\right)\right]=S\left(O_{0}, R_{0}\right) \cup S\left(O, r_{0}\right),
$$

then:

$$
\begin{aligned}
& \int_{\partial\left[B\left(O_{0}, R_{0}\right) \backslash B\left(O, r_{0}\right)\right]} \boldsymbol{V}_{l_{0}} \cdot \boldsymbol{n} \mathrm{d} \sigma \\
& =\int_{M \in S\left(O_{0}, R_{0}\right)} \boldsymbol{V}_{l_{0}} \cdot \boldsymbol{n}_{M} \mathrm{~d} \sigma+\int_{M \in S\left(O, r_{0}\right)} \boldsymbol{V}_{l_{0}} \cdot \boldsymbol{n}_{M} \mathrm{~d} \sigma .
\end{aligned}
$$

2.1 Showing that:

$$
\int_{S\left(O, r_{0}\right)} \boldsymbol{V}_{l_{0}} \cdot \boldsymbol{n}_{C\left(O, r_{0}\right)} \mathrm{d} \sigma=0 .
$$

Indeed: unit outer-normal vector $\boldsymbol{n}_{M}$ relative to domain $B\left(O_{0}, R_{0}\right) \backslash B\left(O, r_{0}\right)$ at arbitrary point $M \in S\left(O, r_{0}\right)$ is $-\boldsymbol{e}_{r}$. This implies:

$$
\boldsymbol{V}_{l_{0}} \cdot \boldsymbol{n}=H(r, \varphi, \theta) \boldsymbol{e}_{\varphi} \cdot\left(-\boldsymbol{e}_{r}\right)=0
$$$$
=\frac{1}{b_{l_{0}}} \int_{0}^{2 \pi} \int_{0}^{\pi} \mathrm{d} \varphi \mathrm{d} \theta \sin \theta Y_{l_{0}}(\theta)\left[f(r, \varphi, \theta) r^{l_{0}+1}(\theta)-a_{l_{0}} r^{2 l_{0}+1}(\theta) Y_{l_{0}}(\theta)\right]-1=0 .
$$

2.2 Showing that:

$$
\int_{S\left(O_{0}, R_{0}\right)} \boldsymbol{V}_{l_{0}} \cdot \boldsymbol{n}_{\partial B\left(O_{0}, R_{0}\right)} \mathrm{d} \sigma=0 .
$$

Indeed: the symmetry of the shape implies that unit outer-normal vector of $S\left(O_{0}, R_{0}\right)$ relative to domain $B\left(O_{0}, R_{0}\right) \backslash B\left(O, r_{0}\right)$ is below in plan generated by the two vectors $\boldsymbol{e}_{r}$ and $\boldsymbol{e}_{\theta}$ which are orthogonal to field vector $\boldsymbol{V}_{l_{0}}$ directed by $\boldsymbol{e}_{\varphi}$. So, we obtain:

$$
\boldsymbol{V}_{l_{0}} \cdot \boldsymbol{n}_{M}=0, \quad \forall M \in S\left(O_{0}, R_{0}\right) .
$$

Then, by inserting (18) and (19) in (9), we deduce that:

$$
\int_{\hat{\partial}\left[B\left(O_{0}, R_{0}\right) \backslash B\left(O, r_{0}\right)\right]} \boldsymbol{V}_{l_{0}} \cdot \boldsymbol{n d} \sigma=0 .
$$

\section{Boundary Equation}

Finally, by inserting (16) and (20) in (10), we obtain that: 
The previous equation ends the proof since it is true for any $l_{0} \in \mathbb{N}$. $\square$

Proposition 3.3. If $f \in W_{2}^{1 / 2}(\partial \Omega) \subset L^{2}(\partial \Omega)$, then problem (P1) have unique solution with the form (7), whose the coefficients are given by:

- $a_{0}=\frac{\left(D W^{-1} r_{0}^{-2}+r_{0}^{-1}\right) \int_{0}^{2 \pi} \int_{0}^{\pi} \mathrm{d} \varphi \mathrm{d} \theta \sin \theta Y_{0}(\theta) f(\varphi, \theta) r(\theta)-c^{*} Y_{0}^{-1}(\theta)}{\left(D W^{-1} r_{0}^{-2}+r_{0}^{-1}\right) \int_{0}^{2 \pi} \int_{0}^{\pi} \mathrm{d} \varphi \mathrm{d} \theta \sin \theta Y_{0}^{2}(\theta) r(\theta)-1}$

- $b_{0}=\frac{\int_{0}^{2 \pi} \int_{0}^{\pi} \mathrm{d} \varphi \mathrm{d} \theta \sin \theta Y_{0}(\theta) r(\theta)\left[f(\varphi, \theta)-c^{*}\right]}{1-\left(D W^{-1} r_{0}^{-2}+r_{0}^{-1}\right) \int_{0}^{2 \pi} \int_{0}^{\pi} \mathrm{d} \varphi \mathrm{d} \theta \sin \theta Y_{0}^{2}(\theta) r(\theta)}$

- $a_{l}=\frac{r_{0}^{l}\left[D(l+1) r_{0}^{-l-1}+W r_{0}^{-l}\right] \int_{0}^{2 \pi} \int_{0}^{\pi} \mathrm{d} \varphi \mathrm{d} \theta \sin \theta Y_{l}(\theta) r^{l+1}(\theta) f(\varphi, \theta)}{r_{0}^{2 l}\left(D l-W r_{0}\right)+\left[D(l+1) r_{0}^{-1}+W\right] \int_{0}^{2 \pi} \int_{0}^{\pi} \sin \theta r^{2 l+1}(\theta) Y_{l}^{2}(\theta) \mathrm{d} \varphi \mathrm{d} \theta}$

- $b_{l}=\frac{r_{0}^{l+2}\left(D r_{0}^{l-1}-W r_{0}^{l}\right) \int_{0}^{2 \pi} \int_{0}^{\pi} \mathrm{d} \varphi \mathrm{d} \theta \sin \theta Y_{l}(\theta) r^{l+1}(\theta) f(\varphi, \theta)}{r_{0}^{2 l+1}\left(D l-W r_{0}\right)+\left[D(l+1)+W r_{0}\right] \int_{0}^{2 \pi} \int_{0}^{\pi} \sin \theta r^{2 l+1}(\theta) Y_{l}^{2}(\theta) \mathrm{d} \varphi \mathrm{d} \theta}$.

where, $r(\theta)=d_{0} \cos \theta+\sqrt{d_{0}^{2} \cos ^{2} \theta+R_{0}^{2}-d_{0}^{2}}$ is the distance $d_{O \rightarrow M}$ between arbitrary point $M$ on sphere $\partial \Omega_{0}=S\left(O_{0}, R_{0}\right)$ and the center $O$.

Proof. It is enough to resolve for any $l \in \mathbb{N}$, the system of two unknowns $a_{l}$ et $b_{l}$ given by the two boundary conditions $(8)$ and $\left(S_{f}\right)$. $\square$

Since the solution of problem (P1) is given from proposition 3.3, then we can deduce its relative Dirichletto-Neumann operator:

Corollary 3.4. The Dirichlet-to-Neumann operator (1) is defined by

$$
\begin{aligned}
\Lambda_{I, \partial \Omega}:\left.f \in W_{2}^{1 / 2}(\partial \Omega) \mapsto \frac{\partial v_{f}}{\partial v}\right|_{\partial \Omega}:=\left.\nabla v_{f}(r, \varphi, \theta) \cdot \boldsymbol{n}_{\partial \Omega}\right|_{\partial \Omega}, \text { where : } \\
\begin{aligned}
\left.\frac{\partial v_{f}}{\partial v}\right|_{\partial \Omega}(\varphi, \theta)= & R_{0}^{-1}\left[r(\theta)-d_{0} \cos \theta\right] \sum_{l=0}^{\infty} \sum_{m=-l}^{m=l}\left[l a_{l} r^{l-1}(\theta)-(l+1) b_{l} r^{-l-2}(\theta)\right] Y_{l, m}(\varphi, \theta) \\
& -R_{0}^{-1} d_{0} \sin \theta \sum_{l=0}^{\infty} \sum_{m=-l}^{m=l}\left[a_{l} r^{l}(\theta)+b_{l} r^{-l-1}(\theta)\right] \partial_{\theta} Y_{l, m}(\varphi, \theta) .
\end{aligned}
\end{aligned}
$$

Here, the coefficients $\left\{a_{l}, b_{l}\right\}_{l \in \mathbb{N}}$ are given by proposition 3.3.

Proof. Since we have that $\boldsymbol{e}_{z}=\cos \theta \boldsymbol{e}_{r}-\sin \theta \boldsymbol{e}_{\theta}$, then unit outer-normal vector $\boldsymbol{n}_{M}$ for arbitrary point $M \in \partial \Omega=S\left(O_{0}, R_{0}\right)$ is given by:

$$
\boldsymbol{n}_{M}=R_{0}^{-1}\left(r_{M} \boldsymbol{e}_{r}-d_{0} \boldsymbol{e}_{z}\right)=R_{0}^{-1}\left\{\left[r(\theta)-d_{0} \cos \theta\right] \boldsymbol{e}_{r}+d_{0} \sin \theta \boldsymbol{e}_{\theta}\right\}
$$

and consequently, this implies:

$$
\begin{aligned}
& \left.\partial_{\nu} v_{f}(r, \varphi, \theta)\right|_{\partial \Omega}:=\left.\nabla v_{f}(r, \varphi, \theta) \cdot \boldsymbol{n}_{M}\right|_{M \in \partial \Omega}=\left.\nabla v_{f}(r, \varphi, \theta) \cdot R_{0}^{-1}\left\{\left[r(\theta)-d_{0} \cos \theta\right] \boldsymbol{e}_{r}+d_{0} \sin \theta \boldsymbol{e}_{\theta}\right\}\right|_{\partial \Omega} \\
& =R_{0}^{-1}\left[r(\theta)-d_{0} \cos \theta\right] \partial_{r} v_{f}(r, \varphi, \theta)-\left.R_{0}^{-1} d_{0} \sin \theta \partial_{\theta} v_{f}(r, \varphi, \theta)\right|_{\partial \Omega} .
\end{aligned}
$$

But, we have from the proof of proposition 3.2 that $r\left(M \in \partial \Omega_{0}\right)=r(\theta)$. Then:

$$
\left.\partial_{v} v_{f}(r, \varphi, \theta)\right|_{\partial \Omega}=R_{0}^{-1}\left[r(\theta)-d_{0} \cos \theta\right] \partial_{r} v_{f}(r(\theta), \varphi, \theta)-R_{0}^{-1} d_{0} \sin \theta \partial_{\theta} v_{f}(r(\theta), \varphi, \theta) .
$$

Consequently, it is enough to replace $v_{f}(r, \varphi, \theta)$ in the previous equation by its value given in ( 7$)$.
Remark 2. For general properties of Dirichlet-toNeumann operators, mainly existence and uniqueness, 
we refer to [10], chapter 4.

Remark 3. Notice that definition of Dirichlet-toNeumann operator (21) implies that it has as eigenfunctions the spherical harmonic function $\left.Y_{l, m}(\theta, \varphi)\right|_{l \in \mathbb{N}, m \leq l l \mid}$, and a discrete spectrum $\sigma_{\Lambda_{I, \Omega \Omega}}:=\left\{\lambda_{l, m}\right\}_{l=0, m \leq l \leq l}^{\infty}$, whose $\lim _{l \rightarrow \infty} \lambda_{l, m}=\infty$.

Corollary 3.5. Dirichlet-to-Neumann operator (21) is unbounded, non-negative, self-adjoint, first-order elliptic pseudo-differential operator with compact resolvent on the Hilbert space $L^{2}(\partial \Omega, \sin \theta \mathrm{d} \varphi \mathrm{d} \theta)$.

Proof. For the proof, we refer to [10], chapter 4 .

Remark 4. Corollary 3.4 implies using Hille-Yosida's theorem that Dirichlet-to-Neumann operator (21) can be generate certain semigroup $\left\{S(t):=\mathrm{e}^{-t \Lambda_{I, \not 2}}\right\}_{t>0}$. Moreover, we can prove using Arzela-Ascoli's criterion that this semigroup is contractant holomorphic in the both Banach space $C(\partial \Omega)$ and $L^{2}(\partial \Omega, \mathrm{d} \theta \sin \theta \mathrm{d} \varphi)$.

Proof. For a complete proof, see [10], chapter 4. 口

\section{Localisation Inverse Problem}

We are interested by resolving the localisation inverse problem of (P1) using the explicit formula of $d_{0}$, which will be calculated in terms of measurable Dirichlet-toNeumann boundary hypothesis on external boundary $\partial \Omega$.

For resolving this problem, we need the following:

i) First, we aim to calculate the total flux $J_{\partial \Omega}$ across external boundary $\partial \Omega$. ii) Second, we aim to find an equation involving the distance $d_{0}:=d_{O \rightarrow O_{0}}$ between $O$ center of cell $\bar{B}$ and $O_{0}$ center of $\Omega$.

Proposition 4.1. The total flux $J_{\partial \Omega}$ and $J_{\partial \mathrm{B}}$ satisfy the following:

$$
J_{\partial \Omega}=J_{\partial B}=2 \sqrt{\pi} b_{0} D .
$$

Proof. Since the differential element at the boundary $\partial B$ and unit outer-normal vector $\boldsymbol{n}_{M}$ at arbitrary point $M \in \partial B$ are respectively equal to $r_{0} \sin \theta \mathrm{d} \varphi \mathrm{d} \theta$ and $\boldsymbol{e}_{r}$, then we deduce from (7) that:

$$
J_{\partial B}:=-D \int_{M \in \partial B} \nabla v \cdot \boldsymbol{n}_{M} \mathrm{~d} \sigma=2 \sqrt{\pi} b_{0} D .
$$

On the other hand, by Gauss-Ostrogradsky theorem, one gets:

$$
\begin{aligned}
J_{\partial \Omega}-J_{\partial B} & :=\int_{\partial \Omega \cup \partial B} \mathrm{~d} \sigma \boldsymbol{j} \cdot \boldsymbol{n} \\
& =-D \int_{\Omega \backslash B} \mathrm{~d} \sigma \nabla \cdot \nabla v \\
& =-D \int_{\Omega \backslash B} \mathrm{~d} V \Delta v=0,
\end{aligned}
$$

where $\mathrm{d} V$ is the volume differential element. Therefore, (22) is deduced.

Since we have, from proposition 4.1, that total flux $J_{\partial \Omega}$ across external boundary $\partial \Omega$ depended only of one coefficient $b_{0}$ of development (7), whose $b_{0}$ depended of distance $d_{0}:=d_{O \rightarrow O_{0}}$, then an equation of $d_{0}:=d_{O \rightarrow O_{0}}$ can be find easily. Indeed:

Corollary 4.2. The distance $d_{0}:=d_{O \rightarrow O_{0}}$ verifies the following equation:

$$
\begin{aligned}
& 1-\left(\frac{D}{W r_{0}^{2}}+\frac{1}{r_{0}}\right) \int_{0}^{2 \pi} \int_{0}^{\pi} \mathrm{d} \varphi \mathrm{d} \theta \sin \theta Y_{0}^{2}(\theta) \sqrt{d_{0}^{2} \cos ^{2} \theta+R_{0}^{2}-d_{0}^{2}} \\
& =\frac{2 \sqrt{\pi} D}{J_{\partial \Omega}} \int_{0}^{2 \pi} \int_{0}^{\pi} \mathrm{d} \varphi \mathrm{d} \theta \sin \theta Y_{0}(\theta) \sqrt{d_{0}^{2} \cos ^{2} \theta+R_{0}^{2}-d_{0}^{2}}\left[f(\varphi, \theta)-c^{*}\right] .
\end{aligned}
$$

Proof. It is enough to insert (22) in the expression of $b_{0}$ given in proposition 3.2 in order to substitute $b_{0}$, after replacing $r(\theta)$ by its value in term of $d_{0}$ :

$$
\begin{aligned}
& 1-\left(\frac{D}{W r_{0}^{2}}+\frac{1}{r_{0}}\right) \int_{0}^{2 \pi} \int_{0}^{\pi} \mathrm{d} \varphi \mathrm{d} \theta \sin \theta Y_{0}^{2}(\theta)\left[d_{0} \cos \theta+\sqrt{d_{0}^{2} \cos ^{2} \theta+R_{0}^{2}-d_{0}^{2}}\right] \\
& =\frac{2 \sqrt{\pi} D}{J_{\partial \Omega}} \int_{0}^{2 \pi} \int_{0}^{\pi} \mathrm{d} \varphi \mathrm{d} \theta \sin \theta Y_{0}(\theta)\left[d_{0} \cos \theta+\sqrt{d_{0}^{2} \cos ^{2} \theta+R_{0}^{2}-d_{0}^{2}}\right]\left[f(\varphi, \theta)-c^{*}\right] .
\end{aligned}
$$

Consequently, the fact that $\int_{0}^{\pi} \mathrm{d} \theta \sin \theta \cos \theta=0$ ends the proof.

\section{Conclusions}

(23) is an equation of the only unknown $d_{0}$ involving the parameters $J_{\partial \Omega}$ and $f:=\left.v\right|_{\partial \Omega}$, which are the Diri-
chlet-to-Neumann hypothesis of problem (P1) on the external boundary, and we can found them from an experimental measures.

To summarize, we have found an equation for $d_{0}$ which is the distance between the center $O$ of the cell $\bar{B}$ and the center $O_{0}$ of $\Omega$, so it remains to find the position of the center $O$. In fact: 
Let $M_{\max }$ and $M_{\min }$ be two points at the external boundary $\partial \Omega$ whose the norm of the local current $\boldsymbol{j}$ reaches respectively its maximum and minimum values, see Figure 1. Then, from the symmetry of the shape, we deduce that the center $O$ of the cell $\bar{B}$ is localized at the line passed by the points $M_{\max }, M_{\min }$ and $O_{0}$, exactly between $M_{\max }$ and $O_{0}$ where the distance $d_{0}$ between $O$ and $O_{0}$ is given by Equation (23).

By conclusion, we can now answer the question posed in the introduction about the uniqueness of the inverse localisation problem for (P1), and we can conclude that total flux $J_{\partial \Omega}$ (2), involving Dirihlet-to-Neumann operator (1), is sufficient to resolve the localisation inverse problem, in three-dimensional case, if the shape is regular. But, it is not enough in other type of inverse problem like geometrical inverse problem, see [5].

\section{Acknowledgements}

I want to thank the CNRS Federation "Francilienne de

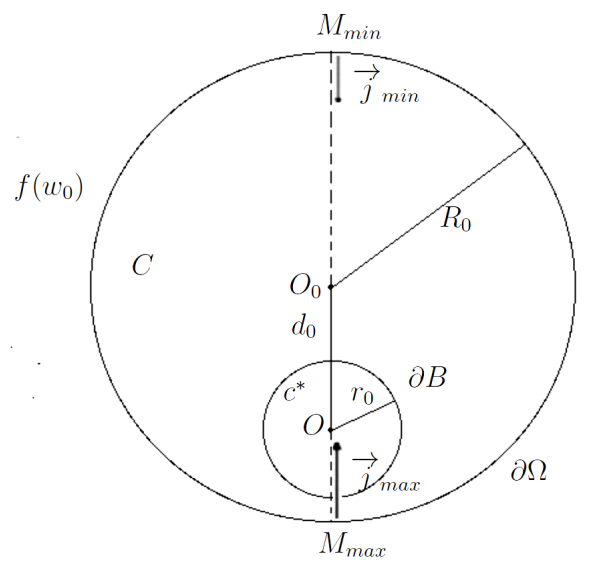

Figure 1. Position of the cell overline $B$.
Mecanique, Materiaux Structures et Procedes" CNRS FR2609, in particularly Prof. Didier Clouteau, for financial support in this study

\section{REFERENCES}

[1] J. Lee and G. Uhlmann, Communications on Pure and Applied Mathematics, Vol. 42, 1989, pp. 1097-1112. doi:10.1002/cpa.3160420804

[2] B. Sapoval, Physical Review Letters, Vol. 73, 1994, pp. 3314-3316. doi:10.1103/PhysRevLett.73.3314

[3] D. S. Grebenkov, M. Filoche and B. Sapoval, Physical Revie E, Vol. 73, 2006, Article ID: 021103. doi:10.1103/PhysRevE.73.021103

[4] O. Kellog, "Foundation of Potential Theory," Dover, New York, 1954.

[5] I. Baydoun and V. A. Zagrebnov, Theoretical and Mathematical Physics, Vol. 168, 2011, pp. 1180-1191. doi:10.1007/s11232-011-0097-8

[6] I. Baydoun, Journal of Modern Physics, 2013, in Press, Article ID: 7501125.

[7] M. E. Taylor, "Partial Differential Equations II: Qualitative Studies of Linear Equations," Springer-Verlag, Berlin, 1996. doi:10.1007/978-1-4757-4187-2

[8] J. K. Hunter and B. Nachtergaele, "Applied Analysis," World Scientific, Singapore, 2001.

[9] D. Tataru, Communications in Partial Differential Equations, Vol. 20, 1995, pp. 855-884. doi: $10.1080 / 03605309508821117$

[10] I. Baydoun, Operateurs de Dirichlet-Neumann et leurs applications. Editions universitaires europeennes. 9783-417-9078-1, 2012. 